The Be Phenomenon in Early-Type Stars, IAU Colloquium 175

ASP Conference Series, Vol. 214, 2000

M. A. Smith, H. F. Henrichs, and J. Fabregat, eds.

\title{
Glimpses of Be Binary Evolution
}

\author{
Douglas R. Gies \\ Center for High Angular Resolution Astronomy, Department of Physics \\ and Astronomy, Georgia State University, Atlanta, GA 30303 U.S.A.
}

\begin{abstract}
Models of close binary evolution predict that mass gainers will be spun up to speeds close to the critical rotational velocity while the mass donors will appear as stripped down He stars, white dwarfs, or neutron stars. I argue here that the mass gainers are closely related to the Be stars. I present a list of the known Be binary systems which consists of those with bright, Roche-filling companions and those with faint or undetected companions. Notably absent are $\mathrm{Be}+\mathrm{B}$ systems which are expected if the Be phase is a stage in the life of a single star. We now have the first example of a $\mathrm{Be}+\mathrm{He}$ star system in the binary, $\phi$ Per, and taken together with the well known Be X-ray binaries, there is clear evidence that some fraction of Be stars are created in binaries; whether all such rapid rotators are so formed remains unknown.
\end{abstract}

\section{Introduction}

Twenty four years ago at IAU Symp. No. 70 (honoring Merrill and McLaughlin), the $\mathrm{Be}$ star community wrestled with the idea that the $\mathrm{Be}$ phenomenon was caused by mass accretion in close binary systems. Křiž \& Harmanec (1975; see also Harmanec \& Křiž 1976) had made the bold suggestion that all Be stars were spun up to rapid rotation by mass transfer in a binary system, and they argued that Be disks are formed directly by mass accretion. Plavec (1976) and collaborators (Polidan 1976; Peters 1976) noted that longer period Algol-type binaries (hot gainer plus cool, Roche-filling donor) could accommodate large disks, and the larger system dimensions would mean that fewer systems would be eclipsing binaries: how many of these might appear as "normal" Be stars?

This binary scenario fell from favor as evidence accumulated that most Be stars cannot be in active mass transfer systems. The first evidence came from the discovery of the Be X-ray binary systems (Be + neutron star). Rappaport \& van den Heuvel (1982) proposed an evolutionary scenario in which Be stars are spun-up through binary transfer, and the reduced donor star (if massive enough) subsequently explodes as a supernova leaving a Be + neutron star (NS) system. These are clearly post-mass transfer binaries, and mass transfer can no longer be the explanation for their emission disks. The next blow against the mass transfer model came from direct searches for companions. If most Be stars are active mass gainers, then their Roche-filling companions should be large enough to detect in the near infrared (where the companions contribute a relatively larger fraction of the flux). However, searches for cool companions generally 
found nothing (35 Be stars, Baade 1992; HR 2142, Waters, Coté, \& Pols 1991; negative results for $\zeta$ Tau but a successful detection for KX And, Floquet et al. 1989). Baade's (1992) conclusion that 'the explanation of the 'Be phenomenon' should not be sought from binarity" still rings true today. Binary processes may or may not have acted to spin up B stars, but we need to search for mechanisms other than mass transfer to explain the circumstellar disks of Be stars.

The Be phenomenon does appear, however, to require rapid rotation, and mass transfer in a close binary offers a potential means to produce rapid rotation. Here I review the models for binary evolution and show how the predictions compare with observations (in particular, the verification of the $\mathrm{Be}+\mathrm{He}$ star stage). The preliminary results suggest that a significant number of $\mathrm{Be}$ stars were indeed formed through binary processes.

\section{Theory of Close Binary Evolution}

There are many excellent studies of the binary evolutionary scenario for the formation of rapidly rotating B-type stars (Pols et al. 1991; De Loore \& Doom 1992; Vanbeveren, Van Rensbergen, \& De Loore 1998). Numerical models usually begin with a system of two intermediate mass, main sequence stars in a short period orbit $(<10 \mathrm{yr}$ ). The initially more massive star expands to fill its critical Roche lobe (usually assumed to occur during $\mathrm{H}$ shell burning - Case B) and begins mass transfer. The gas accreted causes the mass gainer to spin up, and in some models, as little as $0.1 M_{\odot}$ of accreted gas is sufficient to attain the rotational break-up velocity (Packet 1981). The mass transfer first proceeds rapidly and is followed by a extended phase of slower transfer (van der Linden 1987 ) in which the donor loses most of its envelope. (Binaries with very small initial mass ratios, $M_{2} / M_{1}<0.2$, may suffer an accretion rate which is so rapid that the gainer swells and comes into a contact configuration, followed by a spiral-in and merger.) At the conclusion of the mass transfer phase, the system consists of the stripped down, remnant core of the donor (usually referred to as a He star) and the now rapidly rotating gainer (the assumed Be star). The next stage in the evolution of the system depends on the mass of the remnant. If the He star mass is $>2.2 M_{\odot}$, then it will explode as a supernova (Habets 1986) and form either a $\mathrm{Be}+\mathrm{NS}$ binary or an unbound system (if the kick velocity imparted by asymmetry in the supernova explosion is sufficient). On the other hand, if the He star mass is $<2.2 M_{\odot}$, then the remnant will continue to lose mass by a second phase of mass transfer (Case $\mathrm{BB}$ ) or by stellar wind loss to eventually become a white dwarf (WD) (widening the orbit in the process).

Numerical models of this process (Pols et al. 1991; Portegies Zwart 1995; Lipunov, Postnov, \& Prokhorov 1996; Van Bever \& Vanbeveren 1997) require input parameters describing (1) binary frequency, (2) the distributions of mass, mass ratio, and period, (3) the fraction of mass accreted versus lost to the system, (4) the angular momentum lost, (5) the distribution of kick velocities for supernovae, and (6) the relative lifetimes in the various stages. There are significant uncertainties about all of these parameterizations, and consequently, the model predictions about the numbers and kinds of binary products remain diverse. In general, the models suggest that few systems will be found in the active mass transfer stage (because of its relatively short duration), and the 
numbers of post-mass transfer binaries will depend on the mass of the gainer. Massive gainers will tend to have neutron stars or be disrupted while lower mass gainers will have more He star and white dwarf companions.

The predicted numbers of rapid rotators often fall short of the observed numbers of $\mathrm{Be}$ and $\mathrm{Bn}$ stars: this ratio ranges from $\approx 50 \%$ (Pols et al. 1991) to $5-20 \%$ (Van Bever \& Vanbeveren 1997). Taken at face value, these results suggest that binary processes cannot account for all the rapidly rotating $B$ stars, and that some fraction were presumably born with fast spin. However, the model results are preliminary because they are based on parameterizations that may need revision. The best approach to testing their validity is to compare their predictions with the kinds of Be binaries we actually observe.

\section{Observational Results}

Abt \& Cardona (1984) found that Be and B stars generally have the same binary frequency for long period systems (confirmed in a speckle interferometric survey of Be stars by Mason et al. 1997), but they found a deficiency of Be binaries with periods $<0.1 \mathrm{yr}$. Abt \& Cardona suggest that Be stars are born as rapid rotators and that only those lacking companions can avoid spin down caused by the tidal influence of a close neighbor.

The perceived absence of short-period systems could arguably be due to the practical difficulties of measuring radial velocities in Be stars. The photospheric lines of Be stars are generally broad and shallow, often composites of shell absorption and/or disk emission, and frequently subject to rapid variability. Many of the known Be binaries have distorted orbital velocity curves caused by gas streams or an asymmetric circumstellar envelope (leading to an erroneous distribution of longitude of periastron, the Barr effect; Harmanec 1987a). The binary scenario predicts low mass companions with low amplitude radial velocity curves, and, thus, detection will be difficult (few orbital solutions from optical spectra exist for Be X-ray binaries; Negueruela 1998; Wang \& Gies 1998).

Nevertheless, hard work (particularly by investigators at the Ondrejov Observatory) and the use of a variety of observing techniques has led to the discovery of a number of Be binaries with short periods. Table 1 is a list of known and suspected Be binaries with periods less than 10 yr (Batten, Fletcher, \& MacCarthy 1989; Budding 1984; Kogure \& Suzuki 1986). The selection was based on a spectroscopic definition of Be stars (i.e., non-supergiant, B-type stars with persistent and strong $\mathrm{H} \alpha$ emission). Several groups of stars discussed elsewhere were omitted: (1) Be X-ray binaries (see Coe, these proc.), (2) symbiotic variables (usually long period systems with cool, mass losing companions), (3) Algol systems with transient or weak $\mathrm{H} \alpha$ emission (usually those with periods less than $4.5 \mathrm{~d}$; Peters 1989), (4) W Serpentis binaries (evolved binaries with active mass transfer; Anderson, Pavlovski, \& Piirola 1989; Tarasov, these proc.), and (5) chemically peculiar, He strong stars in binaries (where the $\mathrm{H} \alpha$ emission probably originates in magnetically confined gas; Bolton et al. 1998). I also omitted cases where line-doubling was mistaken for core $\mathrm{H} \alpha$ emission ( $\eta$ Ori, AI Cru), stars with weak $\mathrm{H} \alpha$ emission ( $\nu$ Cen; Hendry \& Bahng 1981), a mistaken case where IR features were attributed to a cool companion (HR 894; Polidan 1976), and systems proposed as binaries based on photometry alone. Table 1 gives 
the HD designation, other name, orbital period, eccentricity, mass function (or $m_{2} \sin ^{3} i$ for double-lined systems) $\left(M_{\odot}\right)$, the spectral classification, and a reference code based on the first three letters of the leading author's name and the year of publication (see the reference section for the corresponding citations given in square brackets). Notes on individual stars follow.

HD 17034.- Olson \& Plavec (1997) find masses of 6.25 and $1.60 M_{\odot}$.

HDE 276247.- Olson et al. (1992) discuss the secular changes in the primary eclipse. Wilson \& Plavec (1988) note the rapid rotation of the gainer.

RS Cep.- Olson \& Etzel (1995) find masses of 2.83 and $0.41 M_{\odot}$.

HD 37453.- Parsons \& Bopp (1993) give only the period.

HD 43246.- Dempsey et al. (1990) suggest that the $\mathrm{H} \alpha$ emission originates around the B star. They present radial velocities for the cool component only.

HD 41511.- Welty \& Wade (1995) argue that this is a not Herbig Ae/Be star.

HD 45910.- Elias et al. (1997) present velocities for the cool component only.

HD 50846.- Peters \& Polidan (1998) discuss the disk and FUV spectrum.

HD 59771.- Parsons \& Bopp (1993) give only the period.

PW Pup.- Parsons \& Bopp (1993) quote the period from Eggen (1983).

HD 97528.- Spectral types are from Etzel (1988).

HD 127208.- Dempsey et al. (1990) suggest that the $\mathrm{H} \alpha$ emission originates around the B star. They present radial velocities for the cool component only. HD 174237.- Richards et al. (2000) derive new SB2 orbital elements and argue that part of the $\mathrm{H} \alpha$ emission forms in a disk around the hot star.

HD 207739.- Griffin et al. (1990) argue that the F-star is probably smaller than its Roche surface so this system may not be a classical Algol-type binary. HD 216200.- Hill et al. (1997) derive masses of 6.5 and $1.0 M_{\odot}$ for the hot and cool component, respectively. Bossi et al. (1998) describe the $\mathrm{H} \alpha$ emission.

HD 218393.- Tarasov, Berdyugina, \& Berdyugin (1998) and Floquet et al. (1995) report on the radial velocity curve of the cool secondary; Stefl et al. (1990) discuss the orbital motion of the hot component and its shell lines.

HD 32990.- The Be status of this star is unclear: Kogure \& Suzuki (1986) find no Balmer emission but Tur, Goraya, \& Sharma (1995) show the star has a near-UV and near-IR flux excess indicating the presence of a disk.

HD 37202.- Harmanec (1984) suspected a G-type companion but no cool secondary was found in near-IR study by Floquet et al. (1989). The quoted spectral type is from Slettebak (1982).

HD 41335.- Peters (1983) suggests the companion is Roche-filling, but Waters et al. (1991) argue that the companion is a subdwarf or white dwarf.

HD 142926.- (V839 Her) Koubský et al. (1997) find no evidence of the low-mass secondary, and they dismiss the possibility that the secondary is Roche-filling. They suggest that the secondary is a hot and rotationally unstable object.

HD 161741.- The light curve and orbital phase variations in the $\mathrm{H} \alpha$ emission and UV wind lines suggest that this is an Algol-type but the secondary spectrum remains undetected (Peters 1999).

HD 162732.- (V744 Her) The orbit was also studied by Harmanec, Koubský, \& Krpata (1974). There is no reported detection of the companion.

HD 200120.- This period was first detected by Tarasov \& Tuominen (1987). HD 4180.- Harmanec (1987b) could not confirm the period proposed by Abt \& Levy (1978), and he cautions that the star may not be a spectroscopic binary. 
Table 1. Be Stars in Binaries $(P<10 \mathrm{yr})$

\begin{tabular}{|c|c|c|c|c|c|c|}
\hline $\mathrm{HD}$ & Name & $P(\mathrm{~d})$ & $e$ & $f(m)$ & Spectral Class. & Ref. \\
\hline \multicolumn{7}{|c|}{ A. Bright Companions (Algols) } \\
\hline 17034 & RY Per & 6.8636 & 0 & & B4: V + F7: II-III & Ols1997 \\
\hline 276247 & RW Per & 13.1989 & 0 & $0.37^{a}$ & B9.6eIV-V +K2III-IV & Wil1988 \\
\hline & RS Cep & 12.42 & $\mathbf{0}$ & 0.06 & B9.7e V + G8 III & Ols1995 \\
\hline 37453 & & 66.7 & $\ldots$ & $\ldots$ & $\mathrm{F} 5 \mathrm{II}+\mathrm{Be}$ & Par1993 \\
\hline 43246 & V395 Aur & 23.176 & 0.02 & $\ldots$ & $\mathrm{F} 8 \mathrm{III}+\mathrm{B} 8 \mathrm{Ve}$ & Dem1990 \\
\hline 41511 & 17 SS Lep & 260.34 & 0.02 & $0.12^{a}$ & $\mathrm{~B} 9 \mathrm{Ve}+\mathrm{M} 4 \mathrm{III}$ & Wel1995 \\
\hline 45910 & AX Mon & 232.775 & 0 & $\ldots$ & $\mathrm{B} 2 \mathrm{IIIe}+\mathrm{K} 0 \mathrm{III}$ & Eli1997 \\
\hline 50846 & AU Mon & 11.113 & 0.06 & $1.8^{a}$ & $\mathrm{~B} 3 \mathrm{Ve}+\mathrm{F} 8 \mathrm{III}$ & Sah1997 \\
\hline 59771 & & 89.9 & $\ldots$ & $\ldots$ & $\mathrm{F} 4 \mathrm{II}+\mathrm{B}$ & Par1993 \\
\hline$\ldots$ & PW Pup & 158.0 & $\ldots$ & $\ldots$ & F2ep Iab + B & Par1993 \\
\hline 97528 & TT Hya & 6.953 & $\mathbf{0}$ & $\cdots$ & $\mathrm{B} 9.5 \mathrm{Ve}+\mathrm{K} 0 \mathrm{III}-\mathrm{IV}$ & Pop1989 \\
\hline 127208 & HL Lib & 24.615 & 0 & $\ldots$ & $\mathrm{G} 6 \mathrm{III}+\mathrm{B} 8.5 \mathrm{Ve}$ & Dem1990 \\
\hline 174237 & CX Dra & 6.6960 & 0.05 & $0.75^{a}$ & $\mathrm{~B} 2.5 \mathrm{Ve}+\mathrm{F} 5 \mathrm{III}$ & Ric2000 \\
\hline 207739 & V1914 Cyg & 140.782 & 0.03 & & $\mathrm{~F} 8+\mathrm{B}$ & Gri1990 \\
\hline 216200 & V360 Lac & 10.0854 & 0.0 & 0.0179 & $\mathrm{~B} 3 \mathrm{e}+\mathrm{F} 9 \mathrm{IV}$ & Hil1997 \\
\hline 218393 & KX And & 38.919 & 0.0 & $\ldots$ & B2 IIIe + G8 II & $\operatorname{Tar} 1998$ \\
\hline \multicolumn{7}{|c|}{ B. Faint or Undetected Companions } \\
\hline 10516 & $\phi$ Per & 126.673 & 0 & $1.09^{a}$ & B0.5 III-Ve + sdO & Gie1998 \\
\hline 23862 & $28 \mathrm{BU} \mathrm{Tau}$ & 218.0 & 0.60 & 0.0024 & B8 IVe var & Kat1996 \\
\hline 32990 & $103 \mathrm{Tau}$ & 58.31 & 0.19 & 0.28 & $\mathrm{~B} 2 \mathrm{Ve}$ & Hil1929 \\
\hline 37202 & $\zeta \mathrm{Tau}$ & 132.974 & 0 & 0.018 & B1 IVe & Har1984 \\
\hline 41335 & HR 2142 & 80.86 & 0 & 0.007 & B1 IV-Ve & Pet1983 \\
\hline 109387 & $\kappa$ Dra & 61.55 & 0 & 0.003 & B6 IIIpe & Juz1991 \\
\hline 142926 & $4 \mathrm{Her}$ & 46.192 & 0 & 0.0025 & B9 pe & Kou1997 \\
\hline 161741 & V393 Sco & 7.7125 & ... & $\ldots$ & B3e & Pet1999 \\
\hline 162732 & $88 \mathrm{Her}$ & 86.724 & 0.15 & 0.0061 & B6-7 IV-Ve & Due1988 \\
\hline 183656 & HR 7415 & 214.75 & 0 & 0.0057 & B6e shell & Kou1989 \\
\hline 200120 & 59 Cyg & 28.17 & 0 & 0.08 & B1.5 Vnne & Riv1999 \\
\hline \multicolumn{7}{|c|}{ C. Possible Binaries } \\
\hline 4180 & $o$ Cas & 1033. & 0.11 & 0.72 & B5 IIIe & Abt1978 \\
\hline 23302 & $17 \mathrm{Tau}$ & 4.292 & 0 & 0.0005 & B6 IIIe & Jar 1989 \\
\hline 23630 & $\eta \mathrm{Tau}$ & 4.135 & 0 & 0.0006 & B7 IIIe & Jar1989 \\
\hline 25940 & $48 \mathrm{Per}$ & 16.596 & 0 & 0.0011 & $\mathrm{~B} 3 \mathrm{Ve}$ & Jar1989 \\
\hline 45542 & $\nu \mathrm{Gem}$ & 40.198 & 0 & 0.033 & B6 IIIe & Jar1989 \\
\hline 45995 & HR 2370 & 5.286 & 0.68 & 0.006 & $\mathrm{~B} 2 \mathrm{IVe}$ & Har1987 \\
\hline 50820 & HR 2577 & 18.61 & $\ldots$ & $\ldots$ & $\mathrm{B} 3 \mathrm{IVe}+\mathrm{K} 2 \mathrm{II}$ & Har1987 \\
\hline 58715 & $\beta \mathrm{CMi}$ & 218.498 & 0.48 & 0.17 & $\mathrm{~B} 8 \mathrm{Ve}$ & Jar1989 \\
\hline 58978 & FY CMa & (12.) & $\ldots$ & $\ldots$ & B0 IV:ep & Pet1988 \\
\hline 148184 & $7 \chi \mathrm{Oph}$ & 34.12 & 0.26 & 0.012 & $\mathrm{~B} 1.5 \mathrm{Ve}$ & Har 1987 \\
\hline 183133 & V4372 Sgr & 48.08 & $\ldots$ & & $\mathrm{B} 2 \mathrm{IVe}$ & Law1994 \\
\hline 203025 & HR 8153 & 5.4136 & 0.11 & 0.035 & B2 IIIe & San1926 \\
\hline 224559 & LQ And & 7.4132 & 0 & 0.0006 & B3-4 IV-Ve & Mat1991 \\
\hline
\end{tabular}

${ }^{a} m_{2} \sin ^{3} i$ (for double-lined solutions). 
HD 25940.- Hubert et al. (1997) discuss rapid variations in profiles of 48 Per; they could not confirm the period or orbit proposed by Jarad et al. (1989).

HD 45542.- Jarad et al. (1989) caution that the orbit maybe spurious. The star is a member of a speckle binary $(P=13.00 \mathrm{yr} ;$ Mason 1997).

HD 58978.- The period is based on UV wind line variations (Peters 1999).

HD 148184.- Harmanec (1987b) rules out a longer period, $P=138.8 \mathrm{~d}$, advocated by Abt \& Levy (1978).

HD 203025.- Sanford (1926) found two periodicities, 5.4136 and $225.44 \mathrm{~d}$, in his radial velocity measurements which he interpreted in a triple star model.

The binary systems appearing in Table 1 are grouped into three categories: the first two are based on the relative brightness of the companion, while the third category contains suspected binaries that warrant confirming observations. The first group includes 16 systems with cool, Roche-filling companions, and these are probably Be stars with accretion disks fed by mass transfer from the companion (Křiž \& Harmanec 1975). Note that in a few cases the companion is an F-supergiant, and there maybe some confusion about whether the $\mathrm{H} \alpha$ emission comes from a disk around the B-star or from the wind of the supergiant. The second group is comprised of 11 binaries with faint or undetected companions which could plausibly include the predicted $\mathrm{Be}+\mathrm{He}, \mathrm{Be}+\mathrm{NS}$, and $\mathrm{Be}+\mathrm{WD}$ systems. These systems generally have the long periods, small eccentricities, and small mass functions that are predicted in the binary model. Furthermore, only HD 161741 is a known eclipsing binary, and thus, the companions are generally small compared to the system dimensions.

The most surprising feature of Table 1 is what is missing: there are no $\mathrm{Be}$ $+\mathrm{B}$ or $\mathrm{Be}+\mathrm{Be}$ binaries in the list. The observed mass ratio distribution peaks near unity among normal O- and B-type spectroscopic binaries (Mason et al. 1998; Abt \& Levy 1978). If the Be stars represented some stage in the evolution of single stars, then we would expect to find many examples of nearly equal mass pairs in which one or both were Be stars. The fact that we find no such systems (even among Be binaries with periods $0.1<P<10 \mathrm{yr}$ where tidal breaking arguments fail) is a strong indication that $\mathrm{Be}$ stars are formed through binary interactions that transform close $\mathrm{B}+\mathrm{B}$ systems into $\mathrm{Be}+$ remnant systems. Only in very long period systems $(P>10 \mathrm{yr})$ can a distant B-star witness the Be formation process unscathed.

We can find examples that seem to fit most of the predicted stages of close binary evolution. The $\mathrm{W}$ Ser class probably represents the first phase of rapid mass transfer. The best known member is $\beta$ Lyr which consists of a $2 M_{\odot}$ B8 II bright component which is transferring mass (at a rate of $10^{-4} M_{\odot} \mathrm{yr}^{-1}$ ) to a $12 M_{\odot}$ star hidden within a rapidly rotating disk (possibly with bipolar jets; Harmanec et al. 1996). The He enrichment and extreme CNO cycling of the donor confirm the dramatic removal of its envelope (Balachandran et al. 1986).

The next, slow mass transfer phase is the likely evolutionary stage for most of the Algol-type systems in the first part of Table 1. Many of these systems display $\mathrm{H} \alpha$ emission from the disk and gas stream. In short period systems $(P<4.5 \mathrm{~d})$, the gas stream directly impacts the gainer, and this process can result in transitory disks with weak $\mathrm{H} \alpha$ emission. The gas stream in longer period systems misses the gainer and forms a persistent but time variable accretion disk (Kaitchuck, Honeycutt, \& Schlegel 1985; Peters 1989; Etzel \& Olson 1993; Olson 
\& Etzel 1995; Richards \& Ratliff 1998). Many of the gainers are rapid rotators (RY Per, Olson \& Plavec 1997; RS Cep, Olson \& Etzel 1995; CX Dra, Richards et al. 2000). Note that my list probably underestimates the true numbers of Algols with Be-type primaries: in addition to the omission of the short period, direct impactors, I have also dropped several long period Algols where I could find no reference to $\mathrm{H} \alpha$ emission (TU Mon, W Del), and there are probably systems with Be-type primaries that have been classified as later A-types because of the shell line features formed in their extensive disks (DN Ori, RY Gem, AD Her).

A second stage of rapid mass transfer (case $\mathrm{BB}$ ) is probably occurring in hydrogen deficient binaries like $v$ Sgr which contains a $2.9 M_{\odot}$, A-supergiant orbiting a $4.6 M_{\odot}$ star that is visible only in the UV (Dudley \& Jeffery 1990).

Finally we come to the $\mathrm{Be}+$ remnant systems that maybe partially represented by the systems in the second part of Table 1 . The best evidence for evolved companions to Be stars comes from the study of Be X-ray binaries (Be + neutron star), and some 64 systems are known in the Milky Way (see Coe, these proc.). There are no known $\mathrm{Be}+$ white dwarf binaries (Meurs et al. 1992) despite predictions that many should exist (Waters et al. 1989; Raguzova, these proc.). Negueruela (1998) argues that all the $\mathrm{Be} \mathrm{X}$-ray binaries are $\mathrm{Be}+\mathrm{NS}$ and not $\mathrm{Be}+\mathrm{WD}$ systems. However, $\mathrm{Be}+\mathrm{WD}$ binaries are probably very difficult to detect, and only recently have the first white dwarf companions to normal B-stars been discovered through observations with the Extreme Ultraviolet Explorer satellite (y Pup, Vennes, Berghöfer, \& Christian 1997; $\theta$ Hya, Burleigh \& Barstow 1999). No confirmed examples of the Be + He star stage were known until recent FUV spectroscopy with the Hubble Space Telescope led to the discovery of a hot subdwarf orbiting $\phi$ Per, one of the brightest Be stars in the sky, and in the next section I describe the observations of this remarkable binary.

\section{4. $\phi$ Persei}

Poeckert (1981) made the first definitive orbital solution for the Be star, $\phi$ Per $(P=127 \mathrm{~d})$. Poeckert noted the unusual appearance of He II $\lambda 4686$ emission with an anti-phase velocity curve, and he argued that the companion must be a hot object in order to ionize helium. The first direct hint of a hot secondary came from an examination of IUE spectra by Thaller et al. (1995) who used a Doppler tomography algorithm (Bagnuolo et al. 1994) to reconstruct the secondary spectrum from the ensemble of composite spectra. Although the $\mathrm{S} / \mathrm{N}$ $(\approx 12)$ was too poor to detect the lines of the secondary in any individual IUE spectrum, lines of highly ionized species like $\mathrm{Fe} \mathrm{V}$ did appear weakly in the coadded reconstruction. Verification of the high temperature component required higher S/N spectra, and in a subsequent program of Hubble Space Telescope and Goddard High Resolution Spectrograph observations in 1995-6 we successfully detected and separated out this secondary spectrum (Gies et al. 1998).

Radial velocity measurements of the stellar components in the new HST spectra provided the first definitive orbital elements for the hot companion. The Be disk is inclined nearly edge-on to the line of sight (Quirrenbach et al. 1997), and by assuming that the Be star spin and orbital motion axes are parallel, the inclination limit and double-lined orbital solution yield mass estimates of 
$8.9<M_{p} / M_{\odot}<9.6$ and $1.09<M_{s} / M_{\odot}<1.18$ for the Be star and hot subdwarf, respectively. We used synthetic spectra to model the tomographically reconstructed secondary spectrum, and we determined that the subdwarf has $T_{\text {eff }}=53,000$ and $\log g=4.2$, and contributes about $16 \%$ of the FUV flux (much more than in the optical range). The FUV primary star spectrum appears to be normal for its classification (B0.5 III-IVe) and large projected rotational velocity; however, its position in the HR-diagram suggests the star is overluminous for its mass, an indication of large scale mixing (see also Harmanec, these proc.).

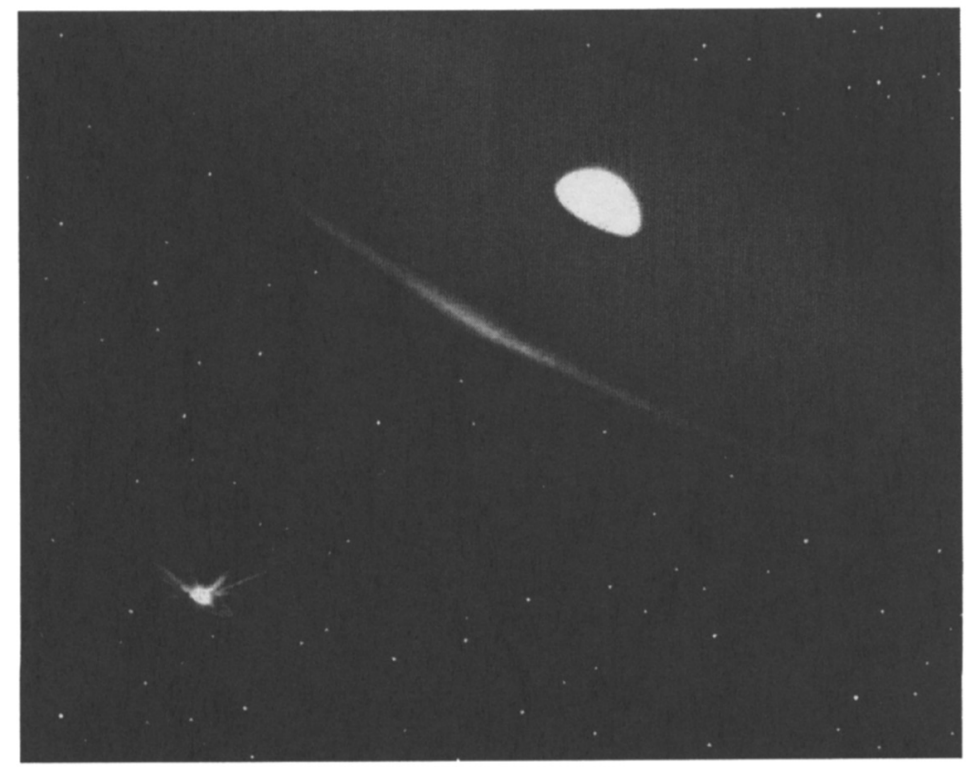

Figure 1. A painting of the $\phi$ Per system (by Bill Pounds).

The HST spectra also revealed the striking development of strong Fe IV absorption lines when the hot subdwarf was in the foreground. The best explanation is that the subdwarf is heating the outer region of the Be star's disk that faces the subdwarf, and that we view the Be star through higher temperature gas at this orbital phase (Fig. 1). Hummel, Štefl, \& Rivinius (these proc.) have demonstrated that the orbital phase variations observed in several optical, He I emission lines also indicate an origin in the heated part of the disk between the stars. This is an important result, because it suggests that such emission line variations can provide an indirect test for the presence of a hot companion.

The HST results strongly support the view that the Be star in $\phi$ Per was spun up to its near critical rotational rate $\left(V \sin i=450 \mathrm{~km} \mathrm{~s}^{-1}\right.$, one of the fastest rotators in the sky) by mass transfer from the now stripped-down, sdO remnant. The hot companion is in fact the brightest hot subdwarf in the sky, but until the HST observations it was lost in the glare of the Be star. There is probably no large scale mass transfer at present, and the Be disk results from outflow from the Be star. 
Vanbeveren et al. (1998) present an evolutionary model for $\phi$ Per that begins with a $6 M_{\odot}+5 M_{\odot}$ pair in a $13.5 \mathrm{~d}$ orbit. They imagine that mass transfer was a conservative process, and shortly after the mass ratio reversed, the system began to widen and the period increase (a trend which continued after mass transfer had ceased due to the stellar wind mass loss of the subdwarf). A comparison of the HR-diagram position of the subdwarf with predicted tracks for post-mass transfer remnants (De Loore \& Vanbeveren 1995) suggests the star's original mass was $\approx 8 M_{\odot}$. This indicates that the mass transfer process was probably non-conservative with a loss of some $2 M_{\odot}$ from the system.

\section{Conclusions}

There are now many examples known of Be stars in the kinds of binary systems predicted by close binary evolution models: (1) $\mathrm{Be}+$ cool, Roche filling companion (Algols), (2) Be + He star ( $\phi$ Per), and (3) Be + neutron star (Be $\mathrm{X}$-ray binaries). The observational evidence clearly shows that rapidly rotating massive stars (the source of Be stars) can be formed through binary processes. It is still unresolved, however, what fraction of the Be population was formed by binary processes versus the fraction born with rapid rotation. Only a small proportion of the Be stars are known binaries (Table 1), but systems with faint companions (white dwarf, He star) are very hard to detect, and, thus, no reliable estimates are presently available about their numbers. Current theoretical models appear to indicate that most Be stars are now truly single objects after disruption of the binary by an asymmetrical kick during the supernova explosion (or became single by mergers in low mass ratio systems).

There are several observational tests ahead that should help us assess the overall importance of the binary route to Be stars. First, new detailed studies of candidate systems with white dwarf or He star companions (see Table 1) are of key importance in establishing the properties and evolutionary paths of the post-mass transfer remnants. Second, there is evidence that Be stars first appear in clusters some $10^{7} \mathrm{yr}$ after birth (Fabregat \& Torrejón 2000). If the binary spin-up process is a major source of Be stars, then we should expect to see the emergence of a rapidly rotating population at that epoch. Investigations of the projected rotational velocity distributions of stars in open clusters would allow us to determine whether or not the rapid rotators first appear at the time when Roche lobe overflow commences in massive close binaries. Finally, the remnants of mass transfer will generally be hot objects with a considerable EUV flux (Pols et al. 1991). In our part of the Galaxy, this part of the spectrum is usually blocked by the interstellar medium, but if a significant number of hot companions do exist, they could contribute to the visible appearance of high redshift galaxies where the EUV flux is Doppler shifted into the optical region.

Acknowledgments. I am grateful for the advice of many colleagues including Gerrie Peters, David McDavid, Petr Harmanec, Paul Etzel, Stéphane Vennes, Myron Smith, and Thomas Rivinius. This work was supported by NASA grant NAG5-2979. Institutional support has been provided from the GSU College of Arts and Sciences and from the Research Program Enhancement fund of the Board of Regents of the University System of Georgia, administered through the GSU Office of the Vice President for Research and Sponsored Programs. 


\section{References}

Abt, H. A., \& Cardona, O. 1984, ApJ, 285, 190

Abt, H. A., \& Levy, S. G. 1978, ApJS, 36, 241 [Abt1978]

Anderson, J., Pavlovski, K., \& Piirola, V. 1989, A\&A, 215, 272

Baade, D. 1992, in Evolutionary Processes in Interacting Binary Stars, ed. Y. Kondo, R. Sistero, \& R. S. Polidan (Dordrecht: Kluwer Academic), 147

Bagnuolo, W. G., Jr., Gies, D. R., Hahula, M. E., Wiemker, R., \& Wiggs, M. S. 1994, ApJ, 423, 446

Balachandran, S., Lambert, D. L., Tomkin, J., \& Parthasarathy, M. 1986, MNRAS, 219, 479

Batten, A. H., Fletcher, J. M., \& MacCarthy, D. G. 1989, Publ. DAO, 17

Bolton, C. T., Harmanec, P., Lyons, R. W., Odell, A. P., \& Pyper, D. M. 1998, A\&A, 337, 183

Bossi, M., Nunez, N. S., Guerrero, G., Sareyan, J.-P., \& Alvarez, M. 1998, A\&AS, 127, 433

Budding, E. 1984, CDS Bull., 27, 91

Burleigh, M. R., \& Barstow, M. A. 1999, A\&A, 341, 795

De Loore, C. W. H., \& Doom, C. 1992, Structure and evolution of single and binary stars (Dordrecht: Kluwer Academic)

De Loore, C., \& Vanbeveren, D. 1995, A\&A, 304, 220

Dempsey, R. C., Parsons, S. B., Bopp, B. W., \& Fekel, F. C. 1990, PASP, 102, 312 [Dem1990]

Dudley, R. E., \& Jeffery, C. S. 1990, MNRAS, 247, 400

Duemmler, R., Kubicela, A., Doazan, V., Bourdonneau, B., \& Arsenijevic, J. 1988, A\&AS, 75, 311 [Due1988]

Eggen, O. J. 1983, AJ, 88, 1676

Elias, N. M., II, Wilson, R. E., Olson, E. C., Aufdenberg, J. P., Guinan, E.,F., Guedel, M., Van Hamme, W. V., \& Stevens, H. L. 1997, ApJ, 484, 394 [Eli1997]

Etzel, P. B. 1988, AJ, 95, 1204

Etzel, P. B., \& Olson, E. C. 1993, AJ, 106, 1200

Fabregat, J., \& Torrejón, J. M. 2000, A\&A, 357, 451

Floquet, M., Hubert, A. M., Chauville, J., Chatzichristou, H., \& Maillard, J.-P. 1989, A\&A, 214, 295

Floquet, M., Hubert, A. M., Hubert, H., Ballereau, D., \& Chauville, J. 1995, A\&A, 294, 227

Gies, D. R., Bagnuolo, W. G., Jr., Ferrara, E. C., Kaye, A. B., Thaller, M. L., Penny, L. R., \& Peters, G. J. 1998, ApJ, 493, 440 [Gie1998]

Griffin, R. F., Parsons, S. B., Dempsey, R., \& Bopp, B. W. 1990, PASP, 102, 535 [Gri1990]

Habets, G. M. J. H. 1986, A\&A, 167, 91

Harmanec, P. 1984, Bull. Astr. Inst. Cz., 35, 164 [Har1984] 
Harmanec, P. 1987a, in Physics of Be Stars, ed. A. Slettebak \& T. P. Snow (Cambridge: Cambridge Univ.), 339

Harmanec, P. 1987b, Bull. Astr. Inst. Cz., 38, 283 [Har1987]

Harmanec, P., Koubský, P., \& Krpata, J. 1974, A\&A, 33, 117

Harmanec, P., \& Křž, S. 1976, in Be and Shell Stars, ed. A. Slettebak (Dordrecht: Reidel), 385

Harmanec, P., et al. 1996, A\&A, 312, 879

Hendry, E. M., \& Bahng, J. D. R. 1981, J. Ap\&A, 2, 141

Hill, G., Harmanec, P., Pavlovski, K., Božić, H., Hadrava, P., Koubský, P., \& Ziznovsky, J. 1997, A\&A, 324, 965 [Hil1997]

Hill, S. N. 1929, Publ. DAO, 4, 261 [Hil1929]

Hubert, A. M., et al. 1997, A\&A, 324, 929

Jarad, M. M., Hilditch, R. W., \& Skillen, I. 1989, MNRAS, 238, 1085 [Jar1989]

Juza, K., Harmanec, P., Hill, G., Tarasov, A. E., Matthews, J. M., Tuominen, I., \& Yang, S. 1991, Bull. Astr. Inst. Cz., 42, 39 [Juz1991]

Kaitchuck, R. H., Honeycutt, B. K., \& Schlegel, E. M. 1985, PASP, 97, 1178

Katahira, J., Hirata, R., Ito, M., Katoh, M., Ballereau, D., \& Chauville, J. 1996, PASJ, 48, 317 [Kat1996]

Kogure, T., \& Suzuki, M. 1986, Ap\&SS, 127, 143

Koubský, P., et al. 1989, Bull. Astr. Inst. Cz., 40, 31 [Kou1989]

Koubský, P., et al. 1997, A\&A, 328, 551 [Kou1997]

Křiž, S., \& Harmanec, P. 1975, Bull. Astr. Inst. Cz., 26, 65

Lawson, W. A., Clark, M., \& Cottrell, P. L. 1994, MNRAS, 266, 740 [Law1994]

Lipunov, V. M., Postnov, K. A., \& Prokhorov, M. E. 1996, A\&A, 310, 489

Mason, B. D. $1997, \mathrm{AJ}, 114,808$

Mason, B. D., ten Brummelaar, T., Gies, D. R., Hartkopf, W. I., \& Thaller, M. L. 1997, AJ, 114, 2112

Mason, B. D., Gies, D. R., Hartkopf, W. I., Bagnuolo, W. G., Jr., ten Brummelaar, T., \& McAlister, H. A. 1998, AJ, 115, 821

Matthews, J. M., Harmanec, P., Walker, G. A. H., Yang, S., \& Wehlau, W. H. 1991, MNRAS, 248, 787 [Mat1991]

Meurs, E. J. A., et al. 1992, A\&A, 265, L41

Negueruela, I. 1998, A\&A, 338, 505

Olson, E. C., \& Etzel, P. B. 1995, AJ, 109, 345 [Ols1995]

Olson, E. C., \& Plavec, M. J. 1997, AJ, 113, 425 [Ols1997]

Olson, E. C., Schaefer, B., Lines, R., Lines, H., \& Fried, R. 1992, AJ, 103, 256

Packet, W. 1981, A\&A, 102, 17

Parsons, S. B., \& Bopp, B. W. 1993, BAAS, 25, 1376 [Par1993]

Peters, G. J. 1976, in Be and Shell Stars, ed. A. Slettebak (Dordrecht: Reidel), 417

Peters, G. J. 1983, PASP, 95, 311 [Pet1983]

Peters, G. J. 1988, ApJ, 331, L33 [Pet1988] 
Peters, G. J. 1989, Space Sci. Rev., 50, 9

Peters, G. J. 1999, private communication [Pet1999]

Peters, G. J., \& Polidan, R. S. 1998, ApJ, 500, L17

Plavec, M. 1976, in Be and Shell Stars, ed. A. Slettebak (Dordrecht: Reidel), 439

Poeckert, R. 1981, PASP, 93, 297

Polidan, R. S. 1976, in Be and Shell Stars, ed. A. Slettebak (Dordrecht: Reidel), 401

Pols, O. R., Coté, J., Waters, L. B. F. M., \& Heise, J. 1991, A\&A, 241, 419

Popper, D. M. 1989, ApJS, 71, 595 [Pop1989]

Portegies Zwart, S. F. 1995, A\&A, 296, 691

Quirrenbach, A., et al. 1997, ApJ, 479, 477

Rappaport, S. A., \& van den Heuvel, E. P. J. 1982, in Be Stars, ed. M. Jaschek \& H. G. Groth (Dordrecht: Reidel), 327

Richards, M. T., Koubský, P., Šimon, V., Peters, G. J., Hirata, R., Škoda, P., \& Masuda, S. 2000, ApJ, 531, 100 [Ric2000]

Richards, M. T., \& Ratliff, M. A. 1998, ApJ, 493, 326

Rivinius, Th., 1999, private communication (see also Rivinius \& Štefl, these proc., p.581) [Riv1999]

Sahade, J., Ferrer, O., Garcia, L. G., Brandi, E., \& Barba, R. 1997, PASP, 109, 1237 [Sah1997]

Sanford, R. F. 1926, ApJ, 64, 172 [San1926]

Slettebak, A. 1982, ApJS, 50, 55

Štefl, S., Harmanec, P., Horn, J., Koubský, P., Kříž, S., Hadrava, P., Božić, H., \& Pavlovski, K. 1990, Bull. Astr. Inst. Cz., 41, 29

Tarasov, A. E., Berdyugina, S. V., \& Berdyugin, A. V. 1998, PAZh, 24, 375 [Tar1998]

Tarasov, A. E., \& Tuominen, I. 1987, Publ. Astr. Inst. Czechosl. Acad. Sci., 70 (10th Euro. Reg. Astr. Meeting - IAU), ed. P. Harmanec, 127

Thaller, M. L., Bagnuolo, W. G., Jr., Gies, D. R., \& Penny, L. R. 1995, ApJ, 448,878

Tur, N. S., Goraya, P. S., \& Sharma, S. D. 1995, PASP, 107, 730

Van Bever, J., \& Vanbeveren, D. 1997, A\&A, 322, 116

Vanbeveren, D., Van Rensbergen, W., \& De Loore, C. 1998, The Brightest Binaries (Dordrecht: Kluwer Academic)

van der Linden, T. J. 1987, A\&A, 178, 170

Vennes, S., Berghöfer, T., \& Christian, D. 1997, ApJ, 491, L85

Wang, Z., \& Gies, D. R. 1998, PASP, 110, 1310

Waters, L. B. F. M., Coté, J., \& Pols, O. R. 1991, A\&A, 250, 437

Waters, L. B. F. M., Pols, O. R., Hogeveen, S. J., Coté, J., \& van den Heuvel, E. P. J. 1989, A\&A, 220, L1

Welty, A. D., \& Wade, R. A. 1995, AJ, 109, 327 [Wel1995]

Wilson, R. E., \& Plavec, M. J. 1988, AJ, 95, 1828 [Wil1988] 


\section{Discussion}

P. Najarro: Do you find consistent results for the stellar radius of $\phi$ Per from the distance, spectroscopy, and binary data? You may have problems with Fe IV line blending around He II $\lambda 1640$ which you used to determine $\log g$.

D. Gies: Using the distance, observed magnitude, model flux ratio, and the mass from the binary orbit, we determine a gravity, $\log g=4.25 \pm 0.17$ which agrees with the value from an analysis of the wings of the He II $\lambda 1640$ line, $\log g=4.2 \pm 0.1$.

P. Najarro: Are your abundance estimates consistent with the evolutionary status?

D. Gies: The subdwarf may be subject to atmospheric diffusion and settling of elements, and so it is difficult make an accurate prediction about the expected abundances. Our analysis is limited, but it appears as though the $\mathrm{C}$ and $\mathrm{O}$ lines are weaker and the $\mathrm{N}$ lines stronger than models based on solar metallicity, so there is some evidence of CNO processed material on the surface of the subdwarf. J. Telting: I have a question related to the kick scenario that is thought to disrupt a would-be Be X-ray binary to leave a single Be star: is there any evidence in the Hipparcos proper motions and/or radial velocities that point to runaway Be stars?

D. Gies: This would be a worthwhile investigation. The runaway velocities are predicted to be some fraction of the Be star's orbital speed before the supernova, and since these semiamplitudes are small $\left(\approx 10 \mathrm{~km} \mathrm{~s}^{-1}\right)$, I expect the Be stars would have small runaway velocities (only applicable to massive Be stars whose companions exploded as a supernova and not to those with He star or white dwarf companions). [After the meeting, Stephen Rinehart examined the Hipparcos data and found that the velocity residuals were similar for $\mathrm{B}$ and $\mathrm{Be}$ stars.]

P. Harmanec: (1) In support of your suggestion that there are probably many more $\mathrm{Be}$ binaries to be discovered, we should note that there are no adequate radial velocity curves for the optical components of Be X-ray binaries. This is probably due to the presence of rapid line profile variations with an amplitude comparable to the orbital radial velocity amplitude.

(2) How do you explain the slow rotation of the secondary of $\phi$ Per?

D. Gies: The secondary probably achieved synchronous rotation during the earlier Roche-filling stage. The current, small $V \sin i$ of the secondary is consistent with synchronous rotation with the orbit, and this may be reasonable if we imagine that the star was a rigid body rotator in which the outer layers were sequentially removed. 